\title{
A Case of Toxic Multinodular Goiter with Anaplastic Carcinoma
}

\author{
Perie Adorable-Wagan, ${ }^{1}$ Audrey Martinez, ${ }^{2}$ Ma.Theresa Chua-Agcaoili, ${ }_{1}$ Elizabeth Ann Samaniego Alcazaren ${ }^{2}$ \\ ${ }^{1}$ Section of Endocrinology and Metabolism, The Medical City Hospital, Pasig City, Philippines \\ ${ }^{2}$ Department of Laboratories, The Medical City Hospital, Pasig City, Philippines
}

\begin{abstract}
This report concerns a 71-year-old female with long standing untreated toxic multinodular goiter (TMG) coexisting with anaplastic thyroid carcinoma who presented with progressive shortness of breath and easy fatigability. Thyroid function tests showed suppressed TSH and elevated FT4 and FT3. Anti-thyroid hormone drugs were started. Thyrotropin receptor antibody was negative. A thyroid scan revealed both hot and cold nodules and fine needle aspiration biopsy (FNAB) of the thyroid gland showed colloid nodule. Chest CT scan imaging revealed an enlarged thyroid gland with the right lobe compressing the trachea. Patient underwent total thyroidectomy, final histopathology showed Anaplastic thyroid carcinoma. Our case is interesting from a pathophysiologic perspective,since it suggests that TMG can potentially transform into an aggressive form of thyroid carcinoma. High index of suspicion in patients with TMG with other risk factors for malignancy requires careful evaluation to detect cancer.
\end{abstract}

Keywords: Anaplastic thyroid carcinoma, Multinodular Toxic Goiter, Hyperthyroidism

\section{Introduction}

Toxic Multinodular goiter (TMG) was previously thought to be a low risk for malignancy compared to solitary thyroid nodule. In a published report, the incidence of malignancy was $9 \%$ in the toxic and $10.58 \%$ in the nontoxic multinodular goiter group. ${ }^{1}$ In a review of resected multinodular goiters by Pedamallu, papillary carcinoma was found to be the most common cancer in $(7 \%){ }^{2}$ However, the finding of anaplastic carcinoma is still very rare. This disease progresses rapidly and treatment should be promply initiated. Only combined multimodal therapy can impact favorably on the local control rate to prevent the high mortality rate from asphyxiation.

\section{Case report}

A frail, 71-year-old female in mild respiratory distress presented at the Emergency Room. Her condition started 2 weeks prior to consult she started to experience shortness of breath and easy fatigability. Pertinent in the history was a goiter which was untreated for several years. There was no noticeable rapid enlargement of the thyroid gland, but there was hoarseness for the past 15 years. She noted weight loss in the last 8 months. She had no prior history of neck radiation, nor family history of goiter. Palpitations and easy fatigability were also present. At the time dyspnea became progressive, patient became weak, and appetite started to decrease. Physical examination showed she was tachycardic, tachypneic, febrile with wheezing and crackles on both lung fields. No palpable thyroid nodules and no cervical lympadenopathy were appreciated. Initial impression was pneumonia with marked leukocytosis at $22.70 \mathrm{mg} / \mathrm{dl}$ with toxic granules. Chest x-ray showed pulmonary nodules and right superior mediastinal mass, inflammatory versus neoplastic in origin. Thyroid function test results showed suppressed TSH $0.01 \mathrm{uIU} / \mathrm{mL}$ (normal $0.35-4.94 \mathrm{uIU} / \mathrm{mL}$ ), FT3 6.37pg/mL (normal 1.45-3.48), and elevated FT4 2.36 ng/dL (normal 0.71-1.85). Patient underwent thyroid scan which showed non-hyperactive multinodular goiter with cold nodules dominant on the right and focal hot areas in both lobes suggestive of goitrous thyroiditis (Figures 1A, 1B, 1C). Thyrotropin receptor antibodies were negative. Ultrasound of the thyroid revealed bilateral thyroid nodules. The right lobe showed three solid nodules with the following measurements: $2.1 \times 1.8 \times 1.10 \mathrm{~cm}$ (with peripheral vascularity), $3.7 \times 3.3 \times 2.0 \mathrm{~cm}$, and $5.0 \times 3.7 \mathrm{~cm}$ (with calcifications and minimal internal and peripheral vascularity). Left lower thyroid lobe had complex nodules with the following measurements: $2.0 \times 2.4 \times 1.8 \mathrm{~cm}$ (predominantly solid) and $4.2 \times 3.3 \times 2.4 \mathrm{~cm}$ (with peripheral vascularity). Treatment for her thyrotoxicosis was started with propylthiouracil $50 \mathrm{mg} / \mathrm{tab} 4$ tabs every 8 hours. With persistent shortness of breath, a chest CT scan with intravenous contrast was done and it showed several
ISSN 0857-1074

Printed in the Philippines

Copyright (C) 2012 by the JAFES

Received March 25, 2012. Accepted April 11, 2012.
Corresponding author: Perie Adorable-Wagan, MD

Section of Endocrinology and Metabolism, Department of Medicine

The Medical City Hospital, Pasig City

Telephone number: +6326356789

Fax number: +6326349396

E-mail address: perie_md@yahoo.com 


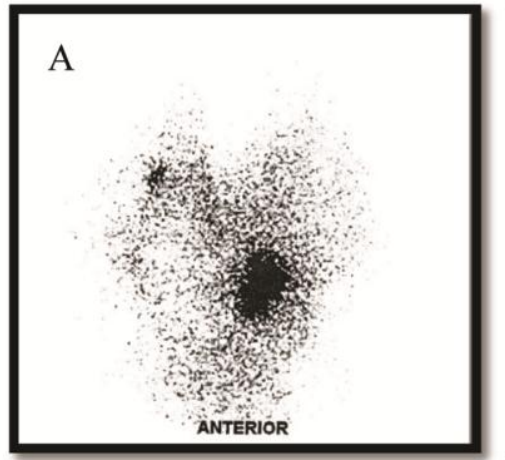

a. Anterior

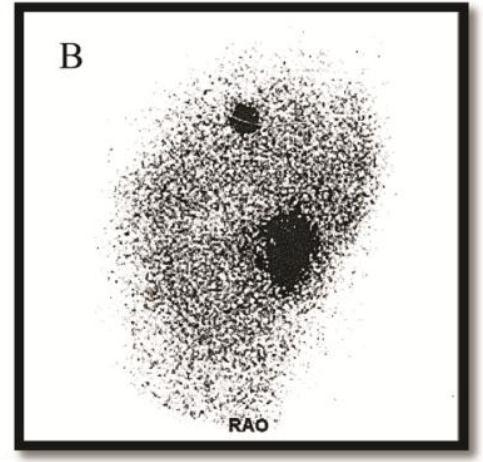

b. Right anterior oblique

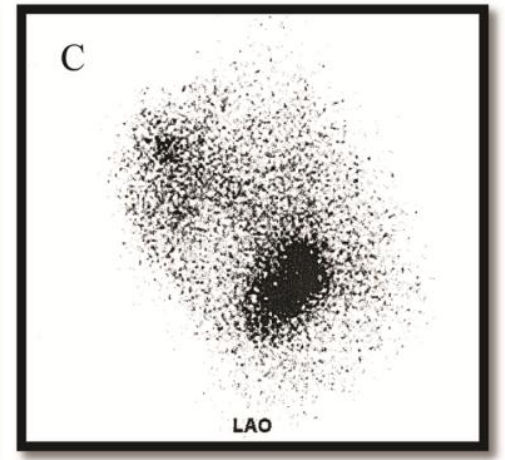

c. Left anterior oblique

Figure 1. Thyroid scan revealed non-hyperactive multinodular goiter with cold nodules dominant on the right and focal hot areas in both lobes. Scintigraphic features suggestive of goitrous thyroiditis.

mildly enhancing, well circumscribed pulmonary nodules in both lung fields. The largest nodule measured $1.5 \mathrm{~cm}$ in its widest diameter, while most of the nodules were subcentimeter in diameter. The enlarged right thyroid lobe spanned an approximate length of $9 \mathrm{~cm}$ and extended into the superior mediastinum. (Figure 2A). The mass compresses the mid segment of the trachea to the left, narrowing its lumen (Figure 2B). After several days of treatment with antibiotics and anti-thyroid medications, the patient had improved and dyspnea had markedly lessened.

An ultrasound guided biopsy of the upper and lower portion of the right thyroid lobe was done and on-site diagnosis showed benign thyrocytes in small follicles and sheets with no nuclear atypia and no cellular overlapping in a background of blood and colloid (The Bethesda System for Reporting Thyroid Cytopathology - Category II). The patient underwent total thyroidectomy.

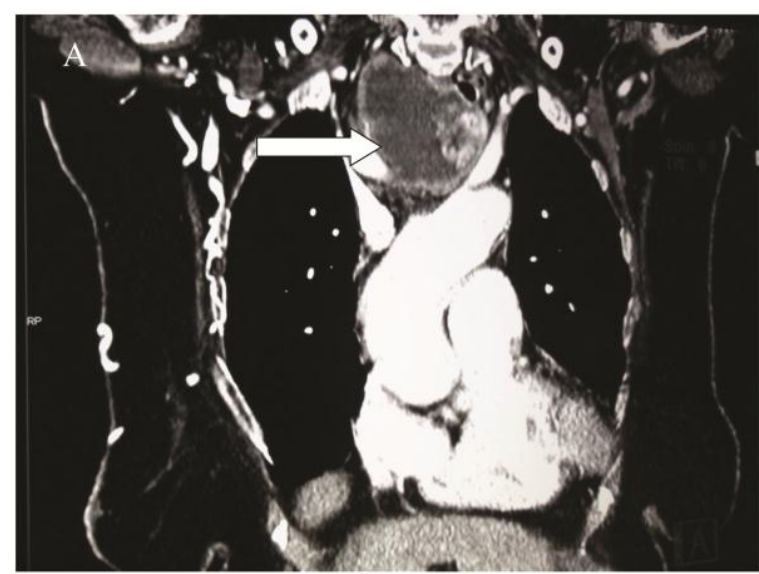

Figure 2A. Coronal view of the chest CT scan.White arrow points to the enlarged thyroid gland with the right lobe demonstrating intrathoracic extension down to the superior mediastinum spanning approximately $9 \mathrm{~cm}$

The right lobe measured $8.3 \times 6.0 \times 4.3 \mathrm{~cm}$, and weighed 119.5 grams with a tan brown surface covered with fibrous tags (Figure 3A). Cut sections showed an ovoid mass, $6.7 \mathrm{x}$ $5.2 \mathrm{~cm}$ in greatest dimensions, with a thin capsule and yellow-tan, gritty to mucoid cut surface with areas of hemorrhage and necrosis. The tumor infiltrated and replaced normal thyroid parenchyma and was surrounded by a thin capsular rim. Tissue sections of the right lobe revealed an infiltrative tumor composed of an admixture of pleomorphic cells, spindle cells and occasional tumor giant cells (Figure 3B). There were small areas showing reminiscent papillary or microfollicle pattern, while some areas showed large epithelioid or multinucleated tumor cells with bizarre nuclei (Figure 3C). Capsular (Figure 3D) and vascular (Figure 3F) invasion were present with extensive tumor necrosis. The uninvolved right and left lobes showed multinodular colloid goiter with lymphocytic thyroiditis. It was signed out as an Anaplastic (undifferentiated) thyroid carcinoma of the right lobe.

Our patient had an uneventful post-operative course. There was marked improvement in her hoarseness and a resolution in her obstructive symptoms. Consult was requested from the Oncology service for planning chemotherapy. She was discharged with orders for readmission after one to two weeks for further treatment. Unfortunately, due to patient's weakened medical condition, she again contracted pneumonia. She was confined in another hospital during this period and due to the severity of her infection, the patient developed respiratory failure and subsequently expired.

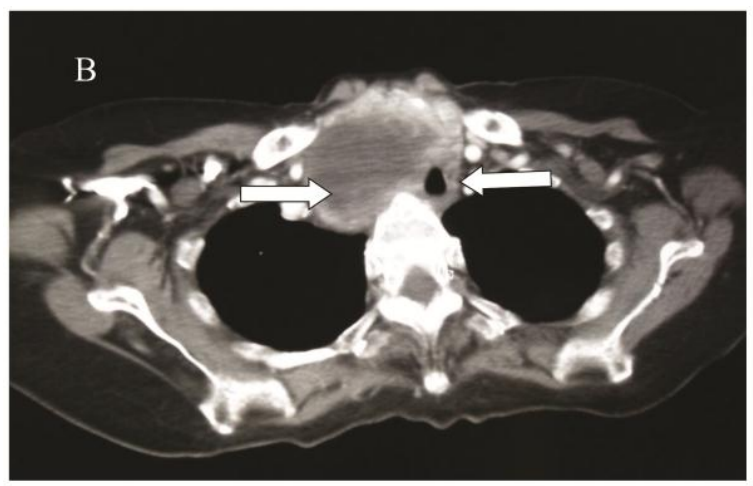

Figure 2B. Axial view of the Chest CT scan. Arrow on the left points to the enlarged right thyroid lobe. Right arrow points to the trachea compressed by the enlarged thyroid gland on the right 


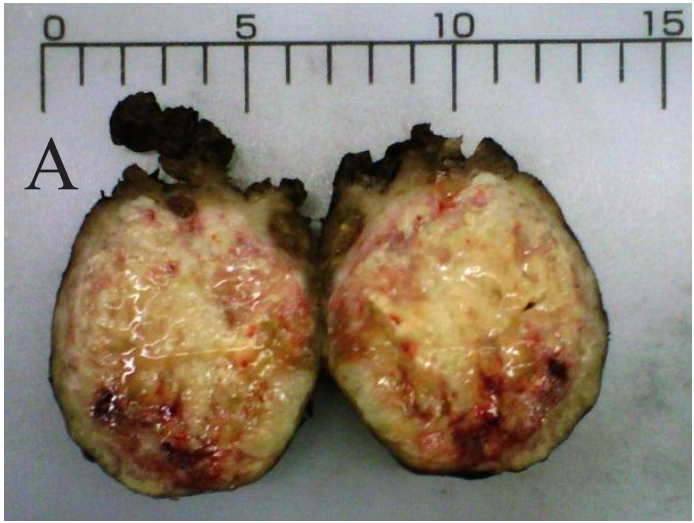

Figure 3A. Tan-yellow, ovoid, thinly encapsulated, infiltrating mass. (6.7 $\times 5.2 \mathrm{~cm}$ in greatest dimensions).

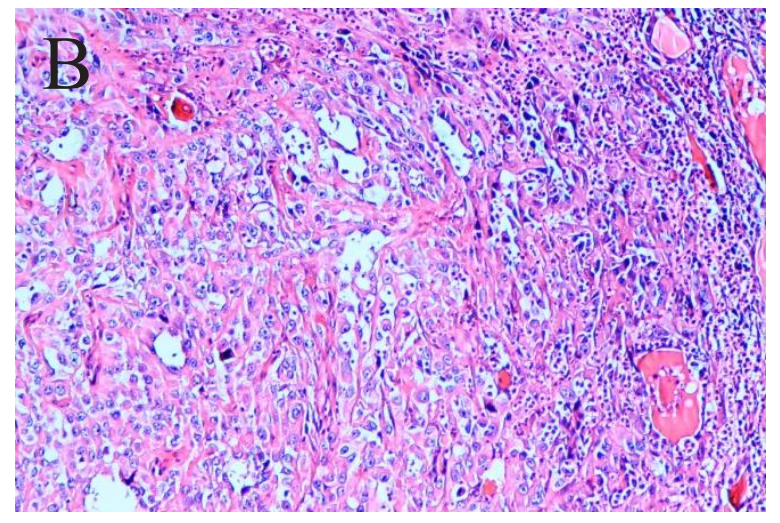

Figure 3B. Undifferentiated tumor. It presents with a sarcomatoid appearance composed of an admixture of spindle and epithelioid cells. (4x and 40x. H\&E.)

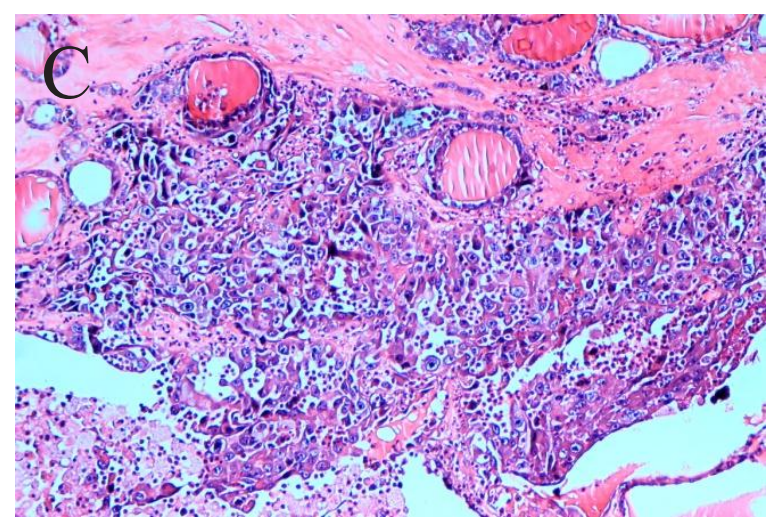

Figure 3C. Infiltrates mostly in solid nests and sheets. (10x. H\&E)

\section{Discussion}

Anaplastic thyroid carcinoma (ATC) rarely presents with thyrotoxicosis. ${ }^{8,16}$ Usual causes of hyperthyroidism with thyroid carcinoma are a coexisting Graves disease or toxic adenoma. These were unlikely in our patient as thyroid ultrasound revealed multinodular goiter and thyrotropin receptor antibody was negative. Thyroid scan showed both hot and cold nodules. With suppressed TSH and elevated thyroid hormones, patient was treated as toxic multinodular goiter (TMG) and treatment with anti-

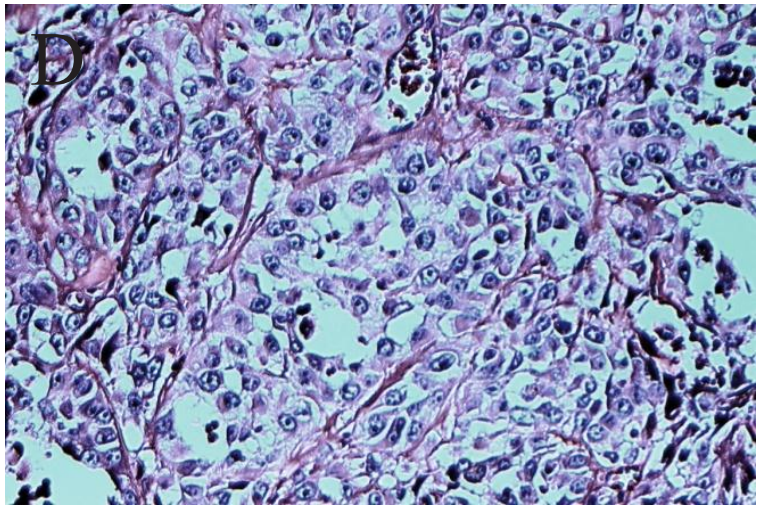

Figure 3D. Epithelioid or multinucleated tumor giant cells with bizarre nuclei. Mitotic figures are present. (10x and 40x. H\&E)

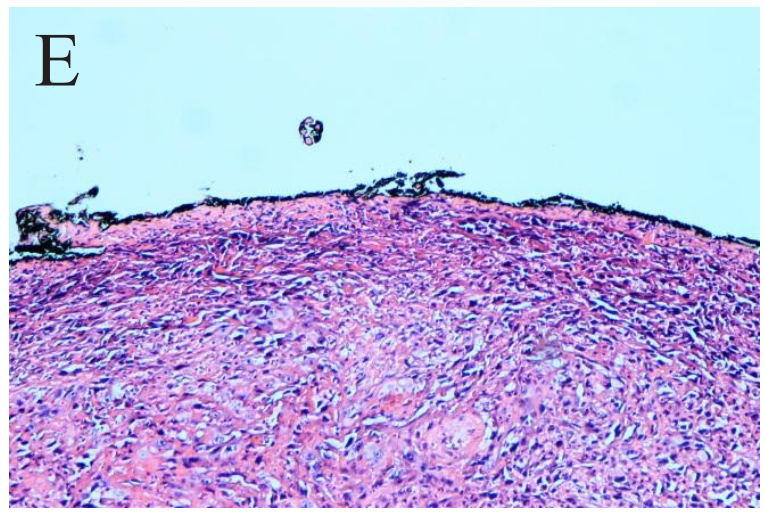

Figure 3E. The tumor grossly and microscopicallly replaces thyroid parenchyma and is sitting on the inked capsule. (4x. H\&E)

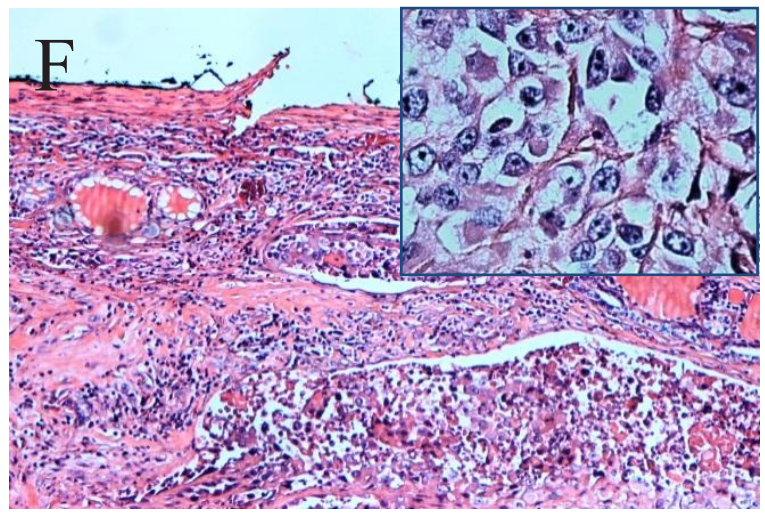

Figure 3F. Vascular invasion is demonstrated. (40x. H\&E)

thyroid hormone showed improvement of symptoms. In a retrospective study done by Memon et $\mathrm{al}^{7}$ (2010), incidence of thyroid carcinoma in multinodular goiter (MNG) varies from $7-13.5 \%$, 1 out of $8(12.5 \%)$ with malignancy showed anaplastic carcinoma. Local data from a retrospective study done by Quiaoit stated 3 out of 400 (0.3\%) thyroid malignancies showed anaplastic thyroid carcinoma. ${ }^{6}$

ATC remains one of the most lethal tumors because of its rapidly growing neck mass. Seventy five percent of patients on presentation have distant metastases, the most 


\begin{tabular}{|c|c|c|c|c|c|}
\hline AUTHORS AND YEAR & AGE & THYROID CA & THYROID FUNCTION TEST & TREATMENT & OUTCOME \\
\hline Oppenheim et al., 1983 & 48 & ATC & $\begin{array}{l}\text { TSH } 2.4 \mathrm{uU} / \mathrm{mL}(\mathrm{n}<10 \mathrm{uU} / \mathrm{mL}) \\
\text { FT3 } 200 \mathrm{ng} / \mathrm{dL} \\
(70-210 \mathrm{ng} / \mathrm{dL}) \\
\text { FT4 19ug/dL } \\
\text { (4-11ug/dL) }\end{array}$ & $\begin{array}{l}\text { Radiotherapy 2,100 rads } \\
\text { Systemic chemotherapy }\end{array}$ & Deceased \\
\hline Villa et al, 2004 & 72 & ATC & $\begin{array}{l}\text { TSH }<0.005 \mathrm{mu} / \mathrm{L} \\
\text { FT3 3.1ng/dL } \\
(0.8-1.55 \mathrm{ng} / \mathrm{dL}) \\
\text { FT4 } 0.47 \mathrm{ng} / \mathrm{dL} \\
(0.28-0.54 \mathrm{ng} / \mathrm{dL})\end{array}$ & Radiotherapy 30Gy & Deceased \\
\hline
\end{tabular}

common site is the lungs which occurs in up to $80 \%{ }^{3,4,9,10}$. The disease contributes to $14-50 \%$ of the mortality with a median survival of 3 to 5 months. 3 ,

Our patient had no discernible anterior neck mass for which no consultation was done until the mass had intrathoracic extension, invaded the trachea and produced compressive symptoms. In a study done by Greenspan et al, ATC reported in the National Cancer Institute's Surveillance, Epidemiology, and End Results (SEER) data base ,only $7.5 \%$ (39 patients out of 516) had an intrathyroidal $\mathrm{ATC}^{5}$. Our patient already had evident lung metastases on chest CT scan.

Anaplastic cells do not express thyroid specific genes and thyroid function test are usually normal. ${ }^{9}$ Sung and Cavalieri explained that hyperthyroidism with thyroid carcinoma include those which produce thyroid hormone autonomously, and causes destructive thyrotoxicosis by rapid cancerous invasion of the normal thyroid tissue. 10,16 Review of literature showed case reports of destructive thyrotoxicosis resulting from the malignant process 8,10 as seen in Table 1. Oppenheim et al.(1983) reported a 48-yearold man with anaplastic thyroid cancer (small cell type) presenting initially with destructive thyrotoxicosis and hypothyroidism. ${ }^{8}$ Villa et al (2004) reported a 76-year-old female diagnosed with Wegener's granulomatosis who presented with thyrotoxicosis in a previously euthyroid multinodular goiter. Histopathological report showed ATC. ${ }^{10}$ Only one report cited ATC with Toxic multinodular goiter. ${ }^{15}$ Schlumberger ${ }^{9}$ stated that over a third of patients with ATC have long standing goiter, while Patel $^{4}$ et al suggested presence of a thyroid tumor may dedifferentiate to ATC. Point mutation in the genes have been identified as an initiating event that lead to transformation of well differentiated thyroid carcinoma to poorly differentiated and anaplastic thyroid carcinoma. One explanation can be due to prolonged stimulation by TSH may be responsible for the transformation into undifferentiated thyroid carcinoma, ${ }^{9}$ though the frequency is rare, since anaplastic thyroid carcinoma represents a small number of percentage of thyroid carcinomas. 5,8 The presence of lymphocytic thyroiditis in our patient with an enlarged thyroid gland may represent chronic autoimmune thyroiditis. Autoimmunity may well coexist with a multinodular goiter and occurs in $10 \%$ of the population with goiter.
Anaplastic thyroid carcinoma is usually far advanced at the time of diagnosis, with extensive local disease and direct invasion of adjacent organs. The options for treatment of ATC include surgery, chemotherapy and radiotherapy. All of these, especially if used alone, most often fail to control local disease. ${ }^{11}$

The diagnosis of ATC is usually suspected on clinical examination, though fine needle aspiration can be an effective diagnostic modality, surgical biopsy as it has been shown to be accurate in $90 \%$ of patients. The initial fine needle aspiration biopsy showed colloid goiter, but sampling error due to increased areas of necrosis, hemorrhage, or fibrosis have been known to occur. Since the clinical suspicion remained high due to obstructive symptoms and weight loss, open biopsy with total thyroidectomy was performed. Final histopathologic report showed anaplastic thyroid carcinoma.

\section{Conclusion}

The incidence of thyroid carcinoma in toxic multinodular goiter is almost negligible, however when they occur, these cancers tend to be more aggressive and are potentially fatal. In the case of our patient, her long standing history of goiter, as well as advanced age were additional risk factors for considering this possibility. In the presence of a rapidly growing and obstructing goiter, and advancing age, it is important to consider malignancy and immediately confirm the diagnosis so appropriate therapy may be instituted.

\section{Potential conflict of Interest None}

\section{References}

1. Cerci C, Cerci SS, et al. Thyroid cancer in toxic and non-toxic multinodular goiter. J Postgrad Med 2007;53:157-160.

2. R. Pedamallu, S. B. Pedamallu, K. V. Rama Rao \& C. Pedamallu Incidence of occult carcinoma in multinodular goiter using histopathological findings. The Internet Journal of Surgery. 2008 Volume 17 Number 1

3. Govardhanan Nagaiah, Akm Hossain,et al.Anaplastic Thyroid Cancer: A Review of epidemiology, pathogenesis, and treatment. Journal of Oncology Volume 2011, Article ID 542358

4. Kepal N. Patel, MD, and Ashok R. Shaha. Poorly differentiated and anaplastic thyroid cancer. April 2006, Vol. 13, No. 2 Cancer Control 119.

5. Kebebew E, Greenspan FS, Clark OH, Woeber KA, McMillan A. Anaplastic thyroid carcinoma: Treatment outcome and prognostic factors. Cancer. 2005 Apr 1;103(7):1330-1335. 
6. Suzette S. Quiaoit, Gabriel V. Jasul Jr, Joselynna A. Quimpo. Thyroid function in thyroid carcinoma: A 5-year retrospective analysis. Phil. J. Internal Medicine. 2009 47: 65-70.

7. Waseem Memon, Tariq Wahab Khanzada, Abdul Samad, Basant Kumar: Incidence of thyroid carcinoma in multinodular goiters. Rawal Medical Journal. 2010.Vol. 35. No. 1

8. Alfred Oppenheim, M.D., Myron Miller,et al. Anaplastic thyroid cancer presenting with hyperthyroidism. The American Journal of Medicine Volume 75. October 1983

9. Schlumberger MJ. Anaplastic Thyroid Carcinoma. Orphanet Encyclopedia. March 2004 ;1-3.

10. Villa ML, Mukherjee JJ, et al. Anaplastic thyroid carcinoma with destructive thyrotoxicosis in a patient with preexisting multinodular goiter. Thyroid 2004;14:227-230

11. Enrico Brignardello, Marco Gallo, et al. Anaplastic thyroid carcinoma: Clinical outcome of 30 consecutive patients referred to a single institution in the past 5 years. European Journal of Endocrinology (2007) 156 425-430.

12. Kyu-Hyoung Lim, Keun-Wook Lee, et al. Anaplastic thyroid carcinoma initially presented with abdominal cutaneous mass and hyperthyroidism. Korean J Intern Med. 2010 December; 25(4): 450453

13. Erik S. Mittra, Ryan D. Niederkohr, et al. Uncommon Causes of Thyrotoxicosis. Nucl Med 2008; 49:265-278

14. Laszlo Hegedu“ S, Steen J. Bonnema, and Finn N. Bennedbæk . Management of simple nodular goiter: Current status and future perspectives. Endocrine Reviews 24(1):102-132

15. Alagöl F, Tanakol R, Boztepe H, et al. Anaplastic thyroid cancer with transient thyrotoxicosis: Case report and literature review. Thyroid. 1999 Oct;9(10):1029-32.

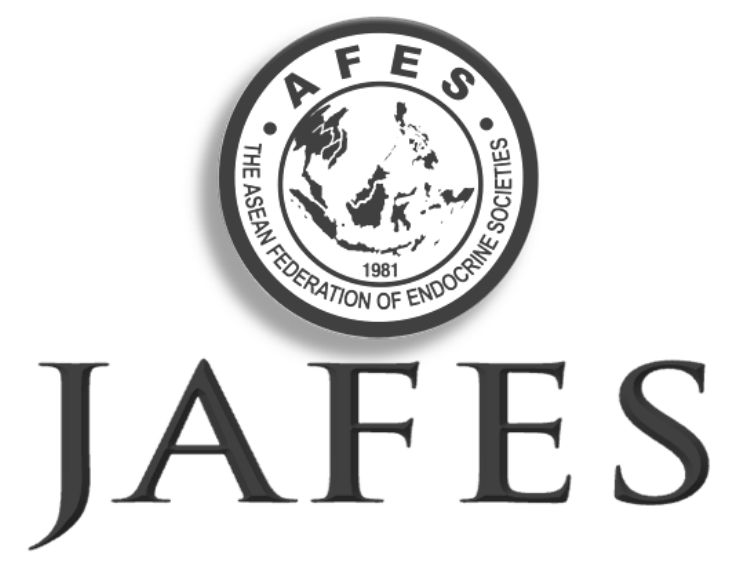

\section{Had an invigorating discussion in Grand Rounds? Share your Clinical Case Seminars at JAFES a Asia.com.}

